\title{
A DIRECT DEDUCTION OF THE CONSTANT PROCESS USED IN THE METHOD OF RIGHT AND WRONG CASES
}

\author{
BY GODFREY H. THOMSON, D.Sc.
}

Armstrong College, Neweas e-upon-Tyne, England

The object of this article is to show how the Constant or Fechner-Müller-Urban Process of calculating thresholds can be directly deduced from first principles. The historical development of the process is first traced, and then contrasted with the direct deduction. The latter throws into clearer relief the nature of the assumptions and approximations made, and justifies Urban's, as against Müller's, Table of Weights.

\section{InTRODUCTION}

The Constant Process is employed to fit the integral of a 'normal' curve to data accumulated by the Method of Right and Wrong Cases. The process has been given various names. Often the name 'Method of Right and Wrong Cases' has been used so as to include not only the method of experimenting but also the above process of dealing with the data after collection. But since many other mathematical processes can be applied to the same data it is better to use a second name. Professor G. E. Müller calls it the 'Konstanzmethode.' Professor Urban speaks of the ' $\Phi(\gamma)$ Hypothesis' and the 'Konstanzmethode," and I have elsewhere called it the 'Constant Process,'s using the word process to indicate a process of calculation, and leaving the word method to mean a method of experimenting. The process can be

1 F. M. Urban, 'Die psychophysischen Massmethoden als Grundlagen empirischer Messungen,' Arehio f. d. ges. Psychol., 16, 1909, p. 366.

'F. M. Urban, 'Die Praxis der Konstanzmethode,' Leipzig, 1912.

'G. H. Thomson, 'A Comparison of Psychophysical Methods,' Brit. Journ. Prychol., 5, 1912, p. 210. 
applied to data collected by other methods, e.g., The Method of Non-Consecutive Groups. ${ }^{1}$

In the descriptions of the Constant Process given by Professor Müller ${ }^{2}$ there is an error of omission which remained undetected until the appearance of Professor Urban's article already quoted, in 1909. Historically the Constant Process arose as an application, to a particular problem, of the Method of Least Squares, of which latter I next give a short account.

\section{The Method of Least Squares}

When observations have given a certain number of observation equations for a smaller number of unknowns, it is impossible in general to find exact solutions for the whole set of equations, for they are, owing to observational errors (and, in psychophysics, also owing to subjective variations) more or less inconsistent with one another. In such a case what is called the Method of Least Squares can be applied to find what are, with certain assumptions, the most probable values of the unknowns. Let there be $k$ equations for $g$ unknowns $(k>g)$, and suppose that these equations are linear, as follows:

$$
\begin{aligned}
& a_{1} x+b_{1} y+c_{1} z+\cdots+f_{1} u+g_{1} w+\alpha_{1}=0, \\
& a_{2} x+b_{2} y+c_{2} z+\cdots+f_{2} u+g_{2} w+\alpha_{2}=0 \text {, } \\
& a_{k} x+b_{k} y+c_{k} z+\cdots+f_{k} a+g_{k} w+\alpha_{k}=0 .
\end{aligned}
$$

Moreover, suppose that these equations are not equally trustworthy, but are based upon various numbers of experiments $n_{1}, n_{2}, \cdots, n_{k}$. Then the rules of the Method of Least Squares run as follows:

To find the 'normal equation' for $x$, multiply each equation (1) by its weight $n$ and (2) by the coefficient of $x$ in that

1 G. H. Thomson, loc. cit., p. 204.

2 G. E. Müller, 'Die Gesichtspunkte und die Tatsachen der psychophysischen Methodik,' Wiesbaden, 1904, par. 11 : 'Ueber die Massbestimmungen des Ortssinnes der Haut mittelst der Methode der richtigen und falschen Fälle,' Pflüger's Archio, Bd. 19, 1879, pp. 191 ff., and elsewhere. 
equation, and then add all the equations together. Similarly, 'normal equations' can be found for $y, z, \cdots, u$ and $w$. There will thus be exactly as many normal equations as unknowns, and the values of the latter found therefrom are the 'most probable' values, provided we assume that the errors or variations arising in our experiment were all 'normally distributed.'

\section{A Description of the Fechner-Müller-Urban Process as it Arose Historically}

This process arose from an application of the above rule to a particular problem in psychophysics. Certain stimuli

$$
r_{1}, r_{2}, r_{3}, \cdots, r_{k}
$$

were presented each $n$ times, ${ }^{1}$ in a certain way, to a subject, who gave at each stimulus value a different proportion of the looked for reaction, these proportions being

$$
p_{1}, p_{2}, p_{3}, \cdots, p_{k} \text {. }
$$

The assumption is now made that

$$
p^{\prime}-1 / 2-\frac{1}{\sqrt{\pi}} \int_{0}^{h(r-S)} e^{-x^{2}} d x=0,
$$

$p^{\prime}$ being a function of which the above $p$ 's are experimental values, while $S$ is the required 'threshold' and $h$ is its 'precision,' $\sigma=\mathrm{I} /(h \sqrt{2})$ being the 'standard deviation' of the distribution. By inserting in equation (I) the $k$ experimental pairs of values of $p$ and $r$ we obtain $k$ observation equations for the two unknowns $S$ and $h$. The equations will never in practice be exactly consistent with each other, and no pair of values $S$ and $h$ will exactly satisfy them all, but will leave small residuals $v_{1}, v_{2}, v_{3}, \cdots, v_{k}$ instead of giving zero in each case.

The Fechner-Müller argument then goes on (erroneously, as Professor Urban showed later) to postulate that the best

1 To avoid unnecessarily complicating the argument I assume each was presented $n$ times. If the different $r$ 's are presented different numbers of times a 'weight' in the ordinary sense of the word has to be attached to each observation equation in what follows, proportional to the number of presentations of that stimulus. 
pair of values $S$ and $h$ is that which makes the sum of the squares of these residuals, $\Sigma \boldsymbol{\Sigma}^{2}$, a minimum. As the rules for forming normal equations are only intended to apply to linear observation equations, however, a plan had to be devised to replace the above equations, which at present include integrals and are anything but linear, by linear equations. This was ingeniously managed by Professor Müller by means of the 'method of weights,' as follows:

In tables of the probability integral we find values of $p$ and corresponding values $\gamma$ of the upper limit of the integral of equation (I). Fechner's Fundamental Table, as it is called, is merely such a table with rather wide intervals specially published for psychologists at that time. We can therefore write down those $k$ values of $\gamma$ which correspond exactly with our $k$ experimental values of $p$, and equate them to the $k$ values obtained by inserting $r_{1}, r_{2}, \cdots, r_{k}$ in the upper limit $h(r-S)$. We thus have $k$ equations of the form

$$
\gamma=h(r-S),
$$

where the $r$ 's are the experimental stimulus values, and the $\gamma$ 's are found (with the aid of a table) from the experimental $p$ 's, and $S$ and $h$ are required. If we write $h S=c$ these become linear equations in $h$ and $c$, viz.,

$$
\gamma-h r+c=0 \text {. }
$$

Any pair of values $h$ and $c$ inserted into the $k$ equations (3) will leave residuals $u_{1}, u_{2}, u_{3}, \cdots, u_{k}$. If we apply the rules of the Method of Least Squares to these equations as they stand, the values of $h$ and $c$ obtained from the normal equations will be those which make $\Sigma u^{2}$ a minimum. This however is not what we want, we want $\Sigma v^{2}$ to be a minimum, the sum of the squares of the residuals of the equations ( $I$ ). We could obtain this if we were to find a set of 'weights' $\Gamma_{1}, \Gamma_{2}, \Gamma_{3}, \cdots, \Gamma_{k}$ to use on the equations (3) such that each $\left(\Gamma u^{2}\right)$ will equal the corresponding $v^{2}$, for the normal equations of the equations (3) thus weighted will then make $\Sigma\left(\Gamma u^{2}\right)$, that is $\Sigma v^{2}$, a minimum. The problem of finding these weights is in effect the problem of finding what change in $p$ 
corresponds to a change $\delta \gamma$ in $\gamma$. We have

$$
p-\frac{1}{2}=\frac{1}{\sqrt{\pi}} \int_{0}^{\gamma} e^{-x^{2}} d x
$$

and therefore

$$
\delta p=\frac{1}{\sqrt{\pi}} \int_{y}^{\gamma+\delta y} e^{-x^{2}} d x .
$$

When $\delta \gamma$ is sufficiently small this integral, which is the area of a narrow element of a curve, may be replaced by the rectangle found by $\delta \gamma$ (width) and $e^{-\gamma^{2}}$ (height), i.e.,

$$
\delta p=\frac{e^{-\gamma^{2}}}{\sqrt{\pi}} \delta \gamma .
$$

$\delta \gamma$ corresponds to a residual $u$, and $\delta p$ corresponds to a residual $v$, so that, since $\Gamma u^{2}$ has to equal $v^{2}$, the sought for weights $\Gamma$ are given by

$$
\Gamma=\frac{e^{-2 \gamma^{2}}}{\pi}
$$

It is convenient that the weights should run from 0 to $I$, and the $\pi$ is therefore omitted in the tabulated values. As weights are merely relative quantities this has no effect on the final result of the calculations. These Müler weights are to be found in several textbooks of experimental psychology. They are however incorrect, or rather incomplete, and should be entirely dropped for Urban's Weights.

Urban's weights differ from Müller's in that they contain further the factor $1 /[4 p(I-p)]$. The need for this factor was overlooked by Professor Müller and was first pointed out by Professor Urban (op. cit., I 1909). It does not arise from the transition from the equations ( $I$ ) to the equations (3), but exists previously to this, because the standard deviation of experimental determinations of a constant proportion $p$ is equal to $\sqrt{n p(\mathbf{1}-p)}$, where $n$ is the number of trials in each experiment, the distribution being binomial in form. If we write

$$
P=\frac{e^{-2 \gamma^{2}}}{4 p(I-p)},
$$

the $P$ 's are Urban's weights which should replace Müller's 
weights. The correctness of Urban's weights is particularly clearly demonstrated in the direct proof of the Constant Process given later in this article.

We have therefore $k$ equations, linear in $h$ and $c$, each with weight $P$, as follows:

$$
\begin{aligned}
& \gamma_{1}-h r_{1}+c=0 \text {, weight } P_{1}, \\
& \gamma_{2}-h r_{2}+c=0 \text {, weight } P_{2}, \\
& \cdot: \cdot \cdot \cdot \cdot \cdot \cdot \cdot \cdot \\
& \cdot \cdot \cdot \cdot \cdot \cdot \\
& \gamma_{k}-h r_{k}+c=0 \text {, weight } P_{k} .
\end{aligned}
$$

By the ordinary rule of the Method of Least Squares the normal equations for $c$ and $h$ are therefore

$$
\begin{aligned}
{[P \gamma]-[P r] h+[P] c } & =0, \\
{[P \gamma r]-\left[P r^{2}\right] h+[P r] c } & =0,
\end{aligned}
$$

where the square brackets are the sign used by Gauss to indicate summation from $\mathbf{I}$ to $k$, traditionally retained in normal equations. From those $h$ and $c$, and therefore $S$, can be calculated.

With this historical development of the Constant Process may now be compared the following direct deduction, where the assumptions and approximations are particularly clearly marked, the rules of the Method of Least Squares are avoided, and Urban's weights are seen to arise from first principles.

\section{Direct Deduction of the Constant Process}

Let there be $k$ different stimuli

$$
r_{1}, r_{2}, r_{3}, \cdots, r_{k}
$$

at each of which a subject is repeatedly tested to ascertain whether or not he reacts in a certain way.

First Assumption.-Assume that for each stimulus r there exists a constant probability $p^{\prime}$ that the reaction in question will occur, so that we have the set of theoretical probabilities

$$
p_{1}{ }^{\prime}, p_{2}{ }^{\prime}, p_{3}{ }^{\prime}, \cdots, p_{k}{ }^{\prime} .
$$


Throughout our argument we shall use dashed letters for theoretical values, undashed letters for observed values.

Now let $n$ experiments be made with each stimulus, and let the proportions of reactions at the respective stimuli be

$$
p_{1}, p_{2}, p_{3}, \cdots, p_{k} \text {. }
$$

What is the probability $W$ of obtaining this particular set of experimental values for the probabilities? The probability of obtaining any $p$ as an experimental value of the corresponding $p^{\prime}$ is, from first principles, equal to

where

$$
\omega=\frac{n !}{(p n) !(q n) !} p^{\prime p n} q^{\prime{ }^{n}}
$$

$$
\begin{aligned}
& q=\mathrm{I}-p, \\
& q^{\prime}=\mathrm{I}-p^{\prime}, \\
& n !=n(n-\mathrm{I})(n-2) \cdots 3,2, \mathrm{I}
\end{aligned}
$$

and since the $n$ experiments made to find one $p$ are quite independent of the $n$ experiments made to find any other $p$, the probability of the simultaneous occurrence of $p_{1}, p_{2}$, $p_{3}, \cdots, p_{k}$ is the product

$$
W=\omega_{1} \omega_{2} \omega_{3} \cdots \omega_{k} .
$$

Now each $p^{\prime}$ must, quite generally, be a function of the stimulus $r$ and of a number of parameters $\alpha, \beta, \gamma, \cdots$. Those values of $\alpha, \beta, \gamma, \cdots$, will be the most probable which make $W$ a maximum, and a necessary condition for this is that

$$
\frac{d W}{d \alpha}=\frac{d W}{d \beta}=\frac{d W}{d \gamma}=\cdots=0 .
$$

These equations are those required to find $\alpha, \beta, \gamma, \cdots$, when the form of the function $p^{\prime}$ has been decided upon. When the value of $W$ is inserted from (I $a$ ) each equation of the system (I2) assumes the form

$$
\sum_{1}^{2} \frac{1}{\omega} \frac{d \omega}{d \alpha}=0
$$

and on further substituting the values of $\omega$ from (II) the sys- 
tem (12) becomes

$$
\sum_{1}^{k} \frac{p-p^{\prime}}{p^{\prime} q^{\prime}} \frac{d p^{\prime}}{d \alpha}=\sum_{1}^{k} \frac{p-p^{\prime}}{p^{\prime} q^{\prime}} \frac{d p^{\prime}}{d \beta}=\cdots=0 .
$$

It is at this point, therefore, that the distinguishing factor of Urban's weights, viz., $I / p^{\prime} q^{\prime}$, makes its appearance. It arises from equation (II) and the assumption which precedes that equation. We have not yet made any assumption as to the relation between $p^{\prime}$ and $r$.

Second Assumption.-Assume now that $p^{\prime}$ is given by

$$
p^{\prime}-1 / 2-\frac{1}{\sqrt{\pi}} \int_{0}^{h(r-S)} e^{-x^{2}} d x=0 .
$$

Thus the parameters are two in number, $S$ and $h$, and we have on differentiating

$$
\begin{gathered}
\frac{d p^{\prime}}{d S}=\frac{-h}{\sqrt{\pi}} e^{-h^{2}(r-S)^{2}}=\frac{-h}{\sqrt{\pi}} e^{-r^{\prime}}, \\
\frac{d p^{\prime}}{d h}=\frac{r-S}{\sqrt{\pi}} e^{-h^{2}(r-S)^{3}}=\frac{r-S}{\sqrt{\pi}} e^{-\gamma^{\prime 2}},
\end{gathered}
$$

where we have written

$$
\gamma^{\prime}=h(r-S)
$$

for abbreviation. From eqn. (13) we therefore have, by substituting for $d p / d S$ and $d p^{\prime} / d h$,

$$
\begin{gathered}
\sum_{1}^{k} \frac{p-p^{\prime}}{p^{\prime} q^{\prime}} h e^{-\gamma^{2}}=0, \\
\sum_{1}^{k} \frac{p-p^{\prime}}{p^{\prime} q^{\prime}}(r-S) e^{-\gamma^{\prime}}=0 .
\end{gathered}
$$

In (15) the $h$ can be brought outside the $\Sigma$ and then dropped: while (16) can be written

$$
\sum_{1}^{l} \frac{p-p^{\prime}}{p^{\prime} q^{\prime}} r e^{-\gamma^{2}}-S \sum_{1}^{h} \frac{p-p^{\prime}}{p^{\prime} q^{\prime}} e^{-\gamma^{\prime 2}}=0,
$$

of which the second term is zero by ( 15$)$. We therefore have as simplified forms of (15) and (16) the equations

$$
\begin{aligned}
& \sum_{1}^{h} \frac{p-p^{\prime}}{p^{\prime} q^{\prime}} e^{-r^{\prime}}=0, \\
& \sum_{1}^{k} \frac{p-p^{\prime}}{p^{\prime} q^{\prime}} r e^{-\gamma^{\prime 2}}=0 .
\end{aligned}
$$


Herein the $p$ 's and $r$ 's are known, and the $p$ "s, $q$ "s, $\gamma^{\prime \prime}$ s are functions of $S$ and $h$ given by (14) and (14a), ( $\left.q^{\prime}=I-p^{\prime}\right)$. These two eqns. (15a) and (16a) are the strict eqns. for finding $S$ and $h$, and $u p$ to this point there are no approximations. A final test of any Gaussian values of $S$ and $h$, in whatever manner found, must be that they give values of $p^{\prime}$, $q^{\prime}$ and $\gamma^{\prime}$ which satisfy ( $\left.15 a\right)$ and (16a).

Except by trial or graphical methods however these equations are not soluble, and approximations are necessary to enable them to be handled. These approximations we now proceed to discuss.

Consider the quantity $p-p^{\prime}$, the difference between the experimental and theoretical values of the probability at the stimulus $r$. From $p$ we can find in tables of the probability integral the value of $\gamma$, the upper limit of the integral, which corresponds to $p$. This $\gamma$ is then an experimental value of the $\gamma^{\prime}$ of eqn. (14a). From $\gamma$ we can in imagination find, if we suppose the $S$ and $h$ known for the moment, a value $r^{\prime}$ from

$$
\gamma=h\left(r^{\prime}-S\right),
$$

$r^{\prime}$ will in general differ from the real value $r$. If we draw a normal curve as in the accompanying diagram, and if $R$ is the position of the stimulus $r$, then the theoretical value $p^{\prime}$ is the area of the curve up to the ordinate at $R$. Let the real value $p$ be (say) greater than this, then $p$ can be repre-

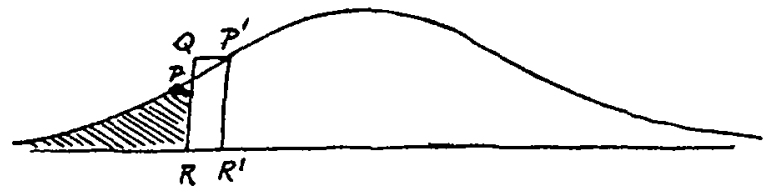

sented as the area to a point a little further on, $R^{\prime}$, corresponding to the value $r^{\prime}$. The quantity $p-p^{\prime}$ is then represented by the area $R P P^{\prime} R^{\prime}$. If this is narrow it may be replaced without great error by the rectangle $R Q P^{\prime} R^{\prime}$, i.e., we write as our first approximation

$$
p-p^{\prime}=\left(r^{\prime}-r\right) e^{-\gamma^{2}},
$$


$e^{-r^{2}}$ being the ord inate $R^{\prime} P^{\prime}$. In doing this we have made our third assumption, viz., that the experimental values $p$ can be sufficiently well fitted to make each $p-p^{\prime}$ small enough to replace by a rectangle. This will for example not be the case if there are gross irregularities, such as 'reversals of the first order,' in the sequence of $p$ 's. The Constant Process should therefore not be applied to such data; if it is, the values obtained from the approximate normal equations (10) will not satisfy the exact equations (15a) and (16a) with sufficient accuracy.

On inserting the value of $p-p^{\prime}$ from eqn. (18) in the eqns. ( $15 a$ ) and $16 a$ ) we obtain

$$
\begin{aligned}
& \sum_{1}^{k} \frac{r-r^{\prime}}{p^{\prime} q^{\prime}} e^{-2 y^{2}}=0, \\
& \sum_{1}^{k} \frac{r-r^{\prime}}{p^{\prime} q^{\prime}} r e^{-2 \gamma^{2}}=0 .
\end{aligned}
$$

Second Approximation.-To further simplify these, we next replace all the theoretical values except $r^{\prime}$ by their observed values, i.e., we write

$$
\begin{aligned}
& \sum_{i}^{l} \frac{r-r^{\prime}}{p q} e^{-2 \gamma^{2}}=0 \\
& \sum_{1}^{b} \frac{r-r^{\prime}}{p q} r e^{-2 \gamma^{2}}=0 .
\end{aligned}
$$

This approximation also rests on the third assumption above. The equations are now the same as the normal equations (I0) as can be seen as follows.

Write as before

and use

$$
P=\frac{e^{-2 \gamma^{2}}}{4 p q}
$$

$$
\gamma=h\left(r^{\prime}-S\right)
$$

to eliminate $r^{\prime}$. We thus obtain

$$
\begin{aligned}
& \sum_{1}^{h} P \frac{h r-\gamma-S h}{h}=0, \\
& \sum_{1}^{h} \operatorname{Pr} \frac{h r-\gamma-S h}{h}=0 .
\end{aligned}
$$


464

GODFREY H. THOMSON

Bring the $1 / h$ outside the summation, drop it, and use the Gauss notation $[x]$ for $\sum_{1}^{k} x$, and we obtain

$$
\begin{aligned}
{[P r] h-[P \gamma]-[P] S h } & =0, \\
{\left[P r^{2}\right] h-\left[P_{r \gamma}\right]-\left[P_{r}\right] S h } & =0,
\end{aligned}
$$

which are identical with our former equations (IO), since $S h=c$. 